\title{
Deep transverse metacarpal ligament: a cadaveric study
}

\author{
Gosavi S. $\mathrm{N}^{1}$., Jadhav S. $\mathrm{D}^{2}$., Zambre B. $\mathrm{R}^{3}$, Vatsala swamy P. ${ }^{4}$ \\ ${ }^{1,2,3}$ Dept. of Anatomy, PDVVPF's Medical College, Ahmednagar/MUHS/India \\ ${ }^{4}$ Dept. of Anatomy, Dr. D. Y. Patil Medical College/ D. Y. Patil/Pune, India
}

\begin{abstract}
The transversely running Deep transverse metacarpal ligament connects the metacarpal head of the index finger to little finger by attachment of volar plate. Sixty cadaveric hands, irrespective of sex, from the department of Anatomy were dissected to study the anatomical features and variation in the attachments of hypothenar muscles. In four out of sixty hands (6.66\%), there was abrupt transition between the volar plate and the fibers of deep transverse metacarpal ligament. While in the rest fifty-six (93.33\%) the transition was smooth. The collateral ligaments were noted on both the sides of the metacarpo phalangeal joints. Insertion of Abductor digiti minimi, flexor digiti minimi brevis in majority cases and opponence digiti minimi in few cases was observed into medial side of the deep transverse metacarpal ligament.
\end{abstract}

Keywords: Deep transverse metacarpal ligament, Hypothenar muscles, Metacarpo phalengeal joint

\section{Introduction}

The strong transverse fibers of the deep transverse metacarpal ligament (DTML) are deep to the palmar aponeurosis and flexor sheaths. They connect the metacarpal head of the index finger to little finger by their attachment with the volar plates.[1] The adjustable position of the first, fourth and fifth metacarpal heads around the fixed second and third form a mobile transverse arch at the level of the metacarpal heads that augments the fixed arch of the distal carpal row.[2] The arches of the hand play an important role during activity; it is their adaptability that enables the hand to adjust to a variety of situations and the transverse arches are maintained by the actions of all the small muscles of the hand.[3] The DTML also contributes to the stability of the mobile arch during grip functions.[4] Considering the importance of trapping of metacarpal head by DTML during reduction of Metacarpophalangeal joints (MCP) joints, this study was carried out with an aim to study the anatomical features of DTML and to note the variation in the insertion of hypothenar muscles into the ligament.

\section{Materials And Method}

Sixty cadaveric hands, irrespective of sex, from the Department of Anatomy, were dissected. After removing the superficial structures, the deep layer of transverse metacarpal ligament was identified. The muscles in the hypothenar region were dissected to see their attachments. The relation of lumbricals, palmar and dorsal interossei and the digital blood vessels and nerves to the deep transverse metacarpal ligament was noted.

\section{Results}

In the cadaveric dissection of sixty hands, thirty of the right and thirty left, the deep transverse metacarpal ligament (DTML) was observed in all the specimens. (Fig.-1) The DTML was seen as transversely running shiny fibers extending from second metacarpal to the fifth metacarpal. The fibrocartilagenous volar plate could be identified between the transversely running fibers. In four out of sixty hands $(6.66 \%)$, there was abrupt transition between the volar plate and the fibers of DTML. While in rest fifty-six (93.33\%) the transition was smooth. The collateral ligaments were noted on both the sides of the metacarpo phalangeal (MCP) joint. The extension of the fibers from DTML to the extensor expansion of the extensor tendons was also noted.

In 70\% (twenty-one of thirty) of the right hands and $76.66 \%$ (twenty-three of thirty) of the left hands the deep fibers of Abductor digiti minimi (AbDM) were inserted into the DTML. In majority of the cases a tendon was present on the deeper surface of the muscle inserting into the DTML.(Fig.-2)

In seven of thirty right (23.33\%) and five of thirty left hands (16.66\%), fibers of Flexor digiti minimi brevis (FDMB) were inserted into the medial side of DTML.(Fig.-2) In five right hands (16.66\%) and in six (20\%) left hands, we observed that, the fibers of AbDM and FDMB were merging with each other and then inserted into the DTML. While in very few i.e. one right and in four left hands the Opponence digiti minimi (ODM) fibers were inserted into the DTML.

\section{Discussion}

Many authors have described DTML as a strong fibrous band which holds the heads of second to fifth metacarpal bones together.[1,2,5,6] The lumbrical muscles lie on volar surface in company with the digital nerves and arteries, which are more volar. The interossei muscle pass on the dorsal side of the deep transverse 
metacarpal ligament.[7] Observations regarding the relations of structures around the DTML in the present study were similar to Kaplan.[7]

The DTML maintains the stability of the transverse metacarpal arch. As the second and fifth metacarpal bones are relatively fixed, movements of the arch are obtained mainly by the medial two metacarpal bones. The role of hypothenar muscles as mobilizers of the metacarpal arch is obvious and is confirmed by the attachments of AbDM and FDMB and the insertion of ODM into the fifth metacarpal. On the other hand, the role of Extensor carpi Ulnaris (ECU) is less obvious but it may well act as a stabilizer of the transverse metacarpal arch. [4]

In all the cadavers observed by AL-Quattan \& Robertson [4], the tendons of the AbDM and /or the FDMB gave slips to the medial aspect of DTML. In one hand there were transverse muscular slips from AbDM inserting into the medial border of the ligament. In the present study also, in majority cases, ( $70 \%$ on right and $76.66 \%$ on left) the $\mathrm{AbDM}$, in the form of tendon from the deeper aspect, was inserting into the medial margin of DTML. In few cases FDMB was also inserting into the DTML directly or through the fibers of AbDM. In one out of thirty right hands and four out of thirty left hands, we observed fibers of ODM, inserting into the DTML. AL-Quattan \& Robertson [4] also observed the insertion of the ODM along the medial margin of the fifth metacarpal bone continuous distally with the DTML.[4]

Origin of AbDM from Flexor carpi ulnaris (FCU) was described by Gray [1] as well as by AL-Quattan \& Robertson [4]. In the present study we observed similar finding in one left hand. Extension of the extensor carpi ulnaris (ECU) tendon to the AbDM and Opponence of the little finger have previously been described [7] but the extension to the transverse metacarpal ligament has not been reported. [4] AL-Quattan \& Robertson [4] observed this in one case. In the present study, a band extending from ECU to the DTML was observed in one right hand.

The palmar (Volar plates) ligaments are unusual. They are thick, dense, fibro-cartilagenous and sited between and connected to the collateral ligaments. They are attached loosely to the metacarpals but firmly to the phalangeal bases. Their palmar aspects are blended with the flexor tendons, whose fibrous sheaths connect with the sides of the groove.[8]

Levangie [2] explained that, the volar plate can be visualized as a fibrocartilage impregnation of the volar portion of the capsule that overlies the metacarpal head. The four volar plates of the MCP joints of the fingers also blend with and are interconnected superficially by the DTML. The volar plate (ligament) at the metacarpo phalangeal joint is a unique structure that increases joint congruence. [2]

The connections of each volar plate to the collateral ligaments of the MCP joint (via the capsule) and the extensor expansion (via the DTML), help stabilize the volar plates on the four metacarpal heads. [4,8-10] The inner surface of the volar plate is effectively a continuation of the articular surface of the base of the proximal phalanx. In extension the plate adds to the amount of surface in contact with the large metacarpal head. Its fibrocartilage composition is consistent with its ability both to resist tensile strength in restricting metacarpophalangeal joint hyperextension and compression forces needed to protect the volar surface of the metacarpal head from the object held in the palm. [11]

Murphy \& Stark [12] explained that, in forced hyperextension of the index finger, usually in a fall on the hand, results in the metacarpal head being pushed through the volar joint capsule. The fibrocartilagenous volar plate is torn loose at its weakest point of attachment - its membranous attachment to the metacarpal. The metacarpal head is displaced volarward, and the volar plate, remaining attached to the phalanx is folded into the joint where it becomes wedged between the metacarpal head and the base of the phalanx.

Kaplan [13] pointed out that there are three obstacles in the reduction of the dislocated MCP joint: wedging of the volar plate within the joint, trapping of the metacarpal head by the palmar fascia and transverse metacarpal ligament, and the displaced flexor tendons which are dorsal to the metacarpal head.

DeCoster et al. [14] noted that the fibrocartilagenous volar plates were inserted into or confluent with the transverse fibers of DTML. In three of eight cases observed by DeCoster et al. [14], there was clear demarcation of this interface but in five of eight, the junction was smooth. In the present study, smooth transition between the volar plate and the fibers of DTML was observed in $93.33 \%$ cases (56 of 60) and in four of sixty cases abrupt transition was seen.

During their study on cadaveric models for dorsal metacarpo-phalangeal (MCP) joint dislocation by forceful hyperextension, DeCoster et al. [14] observed that, in one case, where the interface was clearly demarcated, the volar plate pulled away from the DTML. The volar plate became interposed in the joint without any remaining attachment to the metacarpal, but the DTML remained in its normal volar position. In seven of eight cases, (where the transition was smooth), as the MCP joint was hyperextended, the volar plate pulled the DTML and proximal edge trailed over the metacarpal head. The volar plate and DTML became interposed in the MCP joint. [14] As in the present study, the smooth transition was observed in majority of cases, we can probably presume that in case of dorsal dislocation of MCP joints, chances of volar plate and DTML becoming 
interposed in the joint are more. DeCoster et al. [14] has also stated that, in addition to retraction of volar plate, reduction of DTML to its volar position is necessary to achieve stable, complete reduction of the MCP joint.

\section{Conclusion}

The anatomy of the DTML and the knowledge of relation and insertion of the hypothenar muscles in it will be useful to the hand surgeons while operating on MCP joints.

\section{Referances}

[1] Standring S: Gray's Anatomy- The anatomical basis of clinical practice. $40^{\text {th }}$ edi. (London UK: Elsevier, $2008 ; 877$ )

[2] Levangei PK, Norkin CC. Joint structure and function, a comprehensive analysis. $3^{\text {rd }}$ edi, (India: Jaypee brothers. 2001); $251-89$.

[3] Salter M, Cheshire L. Hand therapy-Principals and Practice. (Ed. Butterwarth Heinemann. 2000;7)

[4] Al-Quattan M, Robertson G. An anatomical study of the deep transverse metacarpal ligament. J Anat. 1993; 12:443-446.

[5] Snell RS: Clinical anatomy by regions. Eighth edi. (New Delhi, India: Lippincott-Williams \& Wilkins, 2008);516-17.

[6] Breathnach AS: Frazer's Anatomy of the Human skeleton. Fifth ed.( London: JS Churchill, 1958);98.

[7] Kaplan EB, Spinner M. Important muscular variations of the hand and forearm. In Spinner M ed,Kaplan's Functional \& surgical anatomy of the hand. Third ed., (Philadelphia: JB Lippincott.1984); 335-49.

[8] Willams P: Gray's Anatomy. 38 ${ }^{\text {th }}$ edi. (New York: Churchill Livingstone, 1995); 472.

[9] Ranney D. The hand as a concept: digital differences and their importance. Clin. Anat. 1995; 8:281-287.

[10] McMinn RMH. Last's anatomy - regional and applied. Eighth ed. UK: Churchill Livingstone, $1990 ; 142$.

[11] Benjamin M, Ralphs JR, Shibu M, Irwin M. Capsular tissues of the proximal interphalangeal joins: normal composition and effects of Dupuytren's disease and rheumatoid arthritis. J Hand Surg 1993; 18B: 370-376.

[12] Murphy AF, Stark HH. Closed dislocation of the metacarpophalangeal joint of the index finger. J Bone and Jt. Surg. 1967; 49-A: 1579-1586.

[13] Kaplan EB. Dorsal dislocation of the metacarpophalangeal joint of the index finger. J Bone and Joint Surg. 1957; 39-A: 1081-1086.

[14] DeCoster TA, McGrew D, Omer GE Jr. Complex dorsal dislocation of the metacarpophalangeal joint: the deep transverse metacarpal ligament as a barrier to reduction. Iowa orthop j. 1988; 8: 9-12.

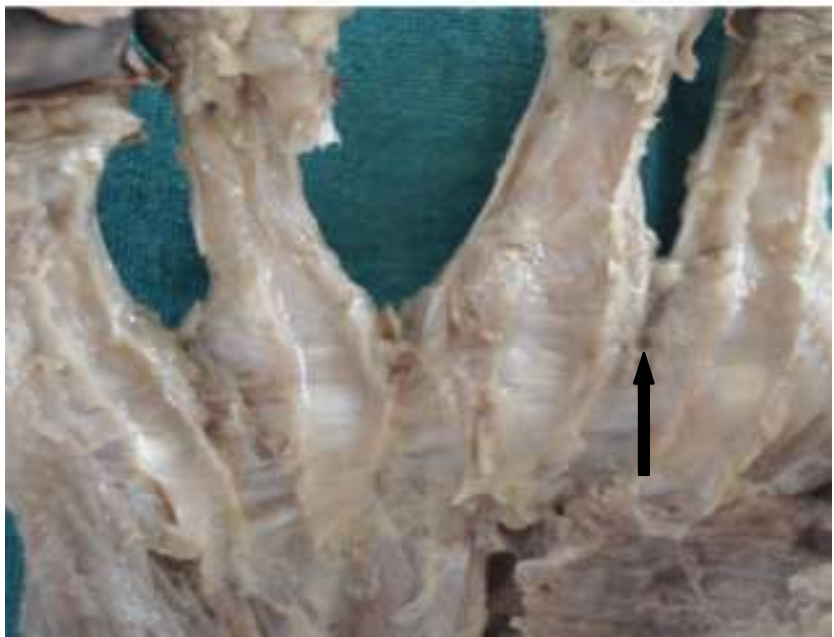

Fig 1: Deep transverse metacarpal ligament Black arrow showing Deep transverse metacarpal ligament

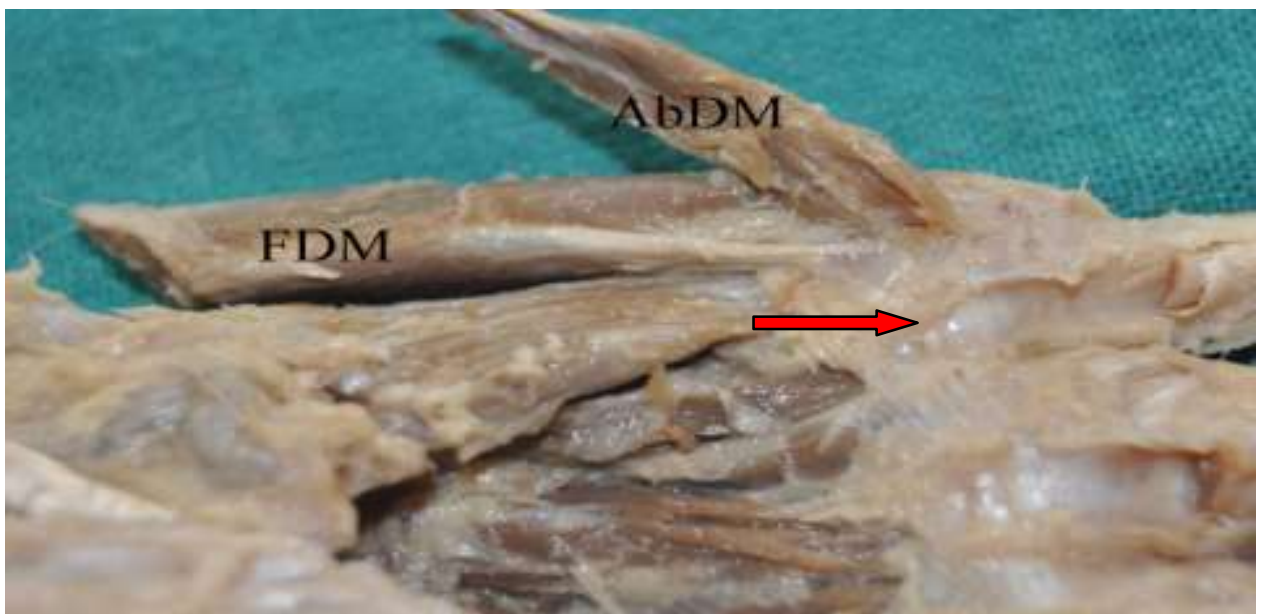

Fig. 2: Photograph showing insertion of Flexor digiti minimi (FDM) and Abductor digiti minimi (AbDM) into the DTML Red arrow indicates fibers of DTML 